\title{
Efetividade de vídeo educativo sobre punção venosa periférica para acadêmicos de enfermagem lusófonos
}

\author{
Effectiveness of educational video on peripheral venous puncture for Portuguese-speaking student nurses
}

Efectividad del video educativo sobre la punción venosa periférica para académicos de enfermería lusófonos

\author{
Beatriz de Sousa Santos' $\mid$; Thamires Sales Macêdo" $\mid \oplus$; Dariane Veríssimo de Araújo" $\mid \oplus$;

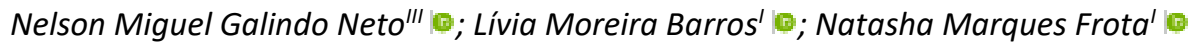

'Universidade da Integração Internacional da Lusofonia Afro-Brasileira, Redenção, CE, Brasil; "Universidade Estadual Vale do Acaraú, Crato, CE, Brasil; "IIInstituto Federal de Educação, Ciência e Tecnologia de Pernambuco, Recife, PE, Brasil

Objetivo: avaliar efetividade de vídeo educativo no conhecimento de acadêmicos de enfermagem lusófonos acerca da punção venosa periférica. Método: estudo quase-experimental, do tipo antes e depois, desenvolvido com 85 acadêmicos de enfermagem, com pré e pós testes realizados a partir de questionário validado composto por 20 questões acerca do conhecimento sobre punção venosa. Resultados: houve melhora significante $(p<0,005)$ nos acertos de sete questões referentes as melhores veias para punção, necessidade de inspeção e palpação, antissepsia em movimentos circulares, posicionamento do torniquete, indicação do scalp, tempo de permanência de dispositivo flexível e complicações do procedimento. No préteste, a mediana de acertos de 15 (Intervalo interquartílico=17), enquanto no pós-teste a mediana de acertos foi de 16 (Intervalo interquartílico=17), $p=0,000$. Conclusão: o vídeo educativo apresenta-se como tecnologia educacional efetiva para auxiliar no ensino da enfermagem sobre a PVP.
\end{abstract}

Descritores: Enfermagem; Alunos de Enfermagem; Tecnologia Educacional; Recursos Audiovisuais; Educação em Saúde.

\section{ABSTRACT}

Objective: to evaluate the effectiveness of an educational video on Portuguese-speaking student nurses' knowledge of peripheral venipuncture. Method: a quasi-experimental study of the before-and-after type was conducted with 85 student nurses by pre- and post-tests using a validated questionnaire of 20 items on vein puncture. Results: correct replies increased significantly $(p<0.005)$ on seven questions regarding: the best veins for puncture, need for inspection and palpation, antisepsis in circular movements, positioning of the tourniquet, indication of scalp use, flexible device dwell time and complications of the procedure. In the pre-test, median hits were 15 (interquartile range $=17$ ), while in the post-test median hits were 16 (interquartile range $=17$ ),$p=0.000$. Conclusion: the educational video was effective educational technology to assist in teaching nurses about peripheral venipuncture.

Descriptors: Nursing; Nursing, Students; Educational Technology; Audiovisual Aids; Health Education.

\section{RESUMEN}

Objetivo: evaluar la efectividad del vídeo educativo sobre el conocimiento de académicos de enfermería lusófonos en cuanto a la punción venosa periférica. Método: un estudio cuasi-experimental, del tipo antes y después, desarrollado junto a 85 estudiantes de la enfermería, con pruebas previas y posteriores realizadas a partir de un cuestionario validado compuesto de 20 preguntas sobre el conocimiento de la punción venosa. Resultados: hubo una mejora significativa $(p<0,005)$ en lo que se refiere a las respuestas correctas a siete preguntas relativas a las mejores venas para la punción, la necesidad de inspección y palpación, la antisepsia en movimientos circulares, la posición del torniquete, la indicación de la aguja mariposa (scalp), el tiempo de permanencia del dispositivo flexible y las complicaciones del procedimiento. En la prueba previa, el promedio de respuestas correctas fue de 15 (rango intercuartil = 17), mientras que en la prueba posterior el promedio de respuestas correctas fue de 16 (rango intercuartil = 17), p=0.000. Conclusión: el vídeo educativo se presenta como una tecnología educativa eficaz para ayudar a enseñar enfermería sobre PVP, punción venosa periférica.

Descriptores: Enfermería; Estudiantes de Enfermería; Tecnología Educacional; Recursos Audiovisuales; Educación em Salud.

\section{INTRODUÇÃO}

O procedimento de punção venosa periférica (PVP) é realizado habitualmente em todo mundo nos serviços de saúde. Estima-se que, nos Estados Unidos, sejam realizadas 200 milhões de punções ao ano, o que configura a PVP como uma das intervenções mais realizadas rotineiramente pela enfermagem no contexto da prática clínica, especialmente no âmbito hospitalar para infusão de fluidos e diversos medicamentos ${ }^{1,2}$.

Sua instalação requer amplo conhecimento técnico-científico para evitar múltiplas tentativas e inserção em locais anatômicos desfavoráveis. É pertinente que o enfermeiro tenha expertise clínica para averiguar o calibre necessário, veias de escolha, funcionalidade e possíveis complicações tendo em vista que o acesso direto na corrente sanguínea possibilita infecções locais e sistêmicas. Desta forma, é indispensável, aos estudantes e profissionais da enfermagem, a aquisição de conhecimentos e habilidades adequados para melhor desempenho nesse procedimento de $\mathrm{PVP}^{3}$. 
O processo de ensino-aprendizagem na graduação em enfermagem tem incorporado diversas estratégias para facilitar a apreensão do conhecimento com destaque para tecnologias de informação e comunicação (TIC), que fornecem subsídio para ampliação do ensino em sala de aula, por meio da utilização de recursos da informática e da telecomunicação ${ }^{4,5}$. Estudo realizado nos Estados Unidos com acadêmicos de enfermagem revelou que vídeo é um dos métodos instrucionais preferidos ${ }^{6}$.

O uso de TICs como vídeos educativos é considerado importante para dinamização do ensino, assim como para o desenvolvimento de competências nos acadêmicos ${ }^{7}$. Ademais, a introdução de recursos tecnológicos viabiliza o rápido acesso à conteúdos e informações, promove responsabilidades, conhecimentos, cientificidade e autonomia ao graduando, não somente dentro da universidade, mas favorece a disponibilidade de métodos de aprendizagem em domicílio ${ }^{7,8}$.

A utilização de recursos tecnológicos, como o vídeo educativo, constitui estratégia complementar para processo de ensino-aprendizagem, pois favorece a aquisição de conhecimentos e habilidades, bem como é uma ferramenta de mídia social que afeta positivamente o processo de ensino e aprendizagem no ensino de enfermagem, que pode corroborar na promoção da autonomia para discentes ${ }^{9}$, além de ser de fácil compreensão e eficaz como recurso pedagógico.

Revisão integrativa realizada no Brasil sobre recursos audiovisuais utilizados no ensino de enfermagem revela que ainda existem poucos estudos na literatura científica sobre a temática, apesar do uso frequente nas aulas de enfermagem para ilustração de procedimentos da prática clínica ${ }^{10}$. Assim, ao considerar que escolha e utilização de tecnologias para o ensino devem ocorrer direcionadas por resultados de pesquisas, aponta-se a necessidade e pertinência de estudos que investiguem a efetividade das variadas opções tecnológicas existentes. Dessa forma, este estudo tem como objetivo avaliar a efetividade do vídeo educativo no conhecimento de acadêmicos de enfermagem lusófonos acerca da pensão venosa periférica.

\section{MÉTODO}

Trata-se de estudo quase-experimental, do tipo antes e depois, que foi desenvolvido no período de fevereiro a dezembro de 2019, com acadêmicos do curso de graduação em enfermagem de uma instituição de ensino superior no estado do Ceará, voltada ao ensino de público luso-brasileiro. A referida instituição possui discentes oriundos dos países que falam língua portuguesa como Brasil, Timor-Leste, Portugal, Cabo Verde, Guiné-Bissau, Angola, Moçambique e São Tomé e Príncipe.

A população-alvo foi representada por discentes matriculados nos semestres 2019.1 e 2019.2 na disciplina de semiotécnica aplicada a enfermagem, na instituição em estudo. Os critérios de inclusão foram: estar regularmente matriculado na disciplina e ter disponibilidade para assistir o vídeo. Como critério de exclusão, discentes que não concluíram o seguimento com pós-teste em 15 dias. Assim, a amostra foi de 85 acadêmicos de enfermagem.

A coleta de dados foi realizada em dois encontros. No primeiro, foi explicado aos participantes o objetivo do estudo e, aos acadêmicos que aceitaram participar, foi solicitado a assinatura do Termo de Consentimento Livre e Esclarecido. Em seguida, houve a aplicação do pré-teste teórico construído e validado conforme orientação de autores ${ }^{11}$, que é composto por 20 questões, do tipo verdadeiro (V) e falso (F), sobre anatomia da rede venosa, material utilizado para PVP, procedimento e complicações. Posteriormente, o vídeo educativo, com duração de sete minutos e 58 segundos, foi projetado por meio do Datashow de forma coletiva para todos os acadêmicos. Vale destacar que, em sala de aula, o vídeo foi exibido somente uma vez, porém foi disponibilizado link na plataforma do YouTube ${ }^{\circledR}$ (https://www.youtube.com/watch?v=tXXHNqhGgIU) com a finalidade de garantir o acesso ilimitado as informações e maior fixação do conteúdo disposto nesta tecnologia digital.

No segundo encontro, realizado após o intervalo de 15 dias da aplicação do pré-teste, os acadêmicos foram reunidos em sala de aula e dispostos em cadeiras enfileiradas para o preenchimento do pós-teste.

Foi elaborado banco de dados no Excel e os dados foram exportados para o software Statistical Package for the Social Sciences (SPSS) 25.0 para a análise estatística. As variáveis categóricas foram analisadas a partir de frequências absolutas e percentuais. A não adesão à normalidade dos dados contínuos foi confirmada a partir do teste de Kolmogorov-Smirnov. Foi utilizado o teste de Mc Nemar para verificar existência de diferenças nos acertos das questões entre pré e pós-teste. A mediana das pontuações totais de acertos antes e após a intervenção educativa foi analisada a partir do teste de Wilcoxon. O nível de significância adotado em todos os testes foi de $5 \%$ e o intervalo de confiança de $95 \%$.

O vídeo foi construído e validado como parte integrante de uma hipermídia educativa sobre Punção Venosa Periférica ${ }^{11}$. No processo de validação, participaram 44 especialistas em que 22 da área da enfermagem e 22 da informática, os quais receberam acesso à hipermídia e, também, aos vídeos incorporados na referida tecnologia. Os itens avaliados foram os objetivos, conteúdo, relevância, efetividade, usabilidade, funcionalidade e eficiência. Especificamente sobre a eficiência dos vídeos, todos os especialistas consideraram esses recursos audiovisuais como adequados e compreensíveis. Após essa etapa, procedeu-se a validação com 21 acadêmicos de enfermagem que 
classificaram a hipermídia e os vídeos disponibilizados como "muito bom" e que poderiam ser utilizados como recurso para facilitar o processo de ensino aprendizagem sobre punção venosa.

O presente estudo seguiu a Resolução 466/2012 que trata de pesquisas com seres humanos do Conselho Nacional de Saúde e foi aprovado pelo Comitê de Ética e Pesquisa da Universidade Federal do Ceará (UFC) com parecer no 666.368 .

\section{RESULTADOS}

Dos 85 participantes, $81,17 \%$ (69) eram do sexo feminino com média de idade de $21,82( \pm 3,32)$. Houve a prevalência da nacionalidade brasileira, sendo que cerca de 82,4\% (70) eram brasileiros, seguida de 5,9\% (5) de São Tomé e Príncipe, 4,7\% (4) de Guiné-Bissau, 2,4\% (2) da Angola, 3,5\% (3) de Cabo-verde, 1,2\% (1) de Timor-Leste. Identificou-se que $84,7 \%$ (72) nunca haviam realizado-acesso venoso periférico. Quanto a experiência com tecnologias educativas, 58,8\% (50) já haviam vivenciado momentos educativos com uso de vídeo. Os dados relacionados aos acertos em cada item do questionário são apresentados na Tabela 1.

TABELA 1: Acertos em cada item do questionário de avaliação do conhecimento teórico dos discentes sobre punção venosa periférica. Redenção, CE, Brasil, 2019.

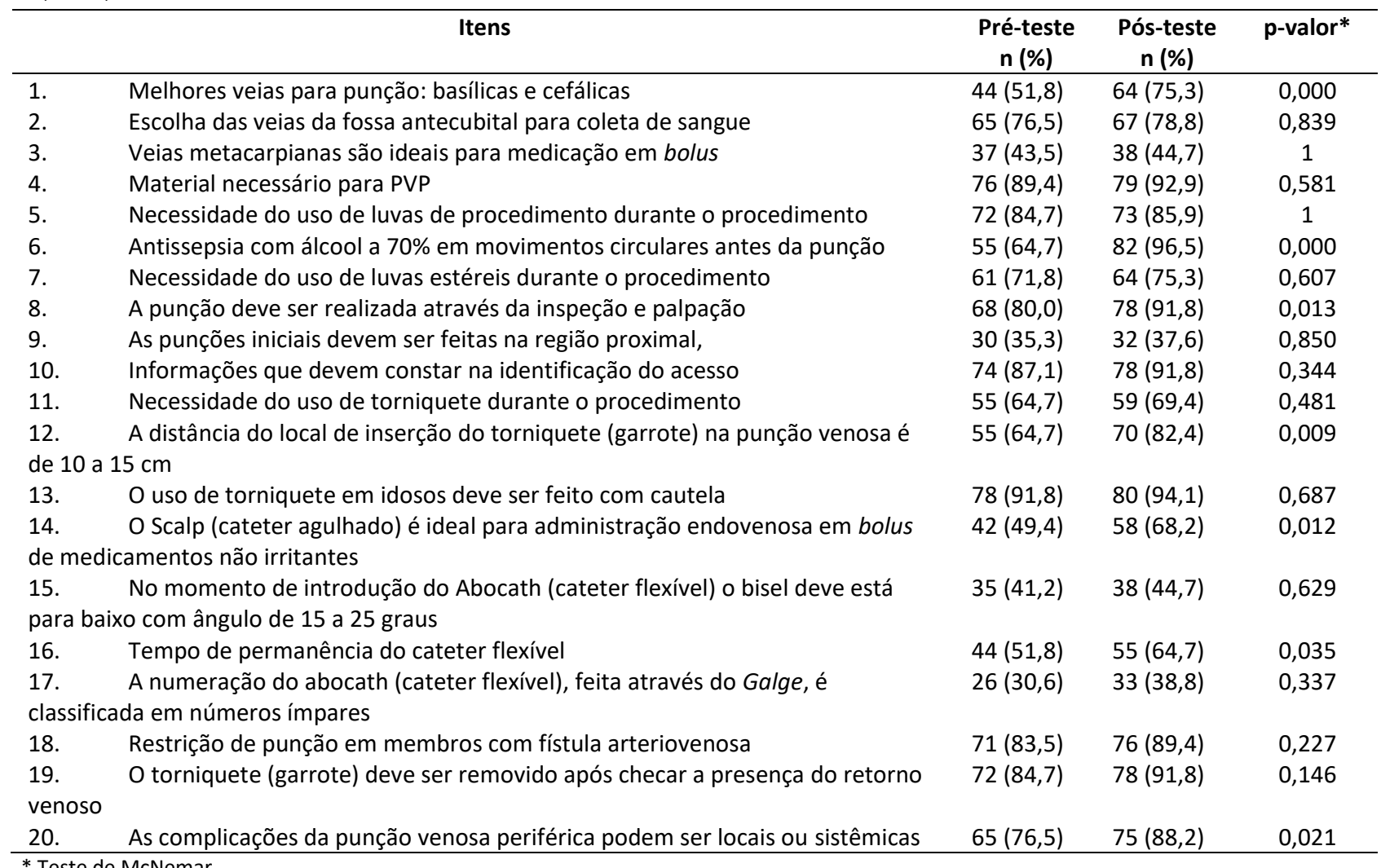

No pré-teste, a mediana de acertos de 15 (Intervalo interquartílico=17), enquanto no pós-teste a mediana de acertos foi de 16 (Intervalo interquartílico=17), p=0,000. A Tabela 1 apresenta os índices de acertos de cada questão que compõem o questionário aplicado.

\section{DISCUSSÃo}

A utilização do vídeo como estratégia educacional demonstra que as tecnologias digitais são ferramentas complementares na graduação em enfermagem proporcionando auxílio no processo de ensino-aprendizado. Neste estudo, a intervenção com vídeo educativo contribuiu para aumento do conhecimento nos itens: seleção de veias $(p=0,000)$, local de punção $(p=0,009)$, antissepsia $(p=0,000)$, inspeção/palpação do local da punção $(p=0,013)$, indicação do uso de scalp $(p=0,012)$, tempo de permanência do cateter $(p=0,035)$ e complicações da punção $(p=0,021)$. 
Estudo realizado em Portugal com 122 acadêmicos de enfermagem revela que os participantes valorizam o uso dos vídeos como tecnologia para favorecer aprimoramento das habilidades nos procedimentos de enfermagem ${ }^{12}$. Na China, estudo de intervenção, que propôs avaliar os benefícios do uso do vídeo no feedback sobre habilidades práticas de 195 acadêmicos de enfermagem, identificou que houve melhora no desempenho em procedimentos como confecção de camas, assepsia, verificação de sinais vitais e oxigenoterapia após recebimento de vídeo por mensagens instantâneas em smartphones ${ }^{13}$.

Portanto, o uso de materiais digitais, como vídeo, nas práticas de ensino, favorece o processo de aprendizagem, tendo em vista que o acesso ao conteúdo pode ser realizado de forma ilimitada, o que oportuniza a repetição das fases do procedimento até compreensão da técnica e resolução de dúvidas. Com isso, o uso desta tecnologia educativa auxilia o estudante como método de aquisição de conhecimentos, especialmente no âmbito da enfermagem ${ }^{14,15}$.

Nesse sentido, o emprego da tecnologia no ensino deve-se ao fato desse recurso contribuir para melhor compreensão do assunto estudado bem como por permitir ao acadêmico a flexibilização do conhecimento com respeito ao ritmo de aprendizagem de cada indivíduo ${ }^{16}$. Ademais, favorece o melhor desempenho dos estudantes diante da possibilita de múltiplas visualizações do vídeo, assim como auxilia no desenvolvimento de competências por meio de ferramentas de informática.

Em concordância a isso, estudo com desenho metodológico realizado em Ribeirão Preto, no interior de São Paulo, sobre utilização de simulador de realidade virtual foi considerado ferramenta válida e promissora para ensinar o procedimento de coleta de sangue a vácuo em adultos entre estudantes de graduação em enfermagem ${ }^{17}$. Na Austrália, estudo qualitativo com 142 acadêmicos de enfermagem revela que o uso de vídeos favoreceu a autoavaliação e reflexão crítica sobre habilidades na comunicação com paciente bem como aumentou a confiança dos discentes em interagir com o público ${ }^{18}$.

Entretanto, na Índia, estudo quase-experimental com objetivo de comparar o uso do vídeo educativo na demonstração da palpação obstétrica com 60 acadêmicos de enfermagem evidenciou que o ensino tradicional em laboratório obteve melhores índices de acertos na avaliação da habilidade quando comparado ao vídeo ${ }^{19}$. Isso demonstra que, apesar do vídeo ser recurso audiovisual fácil de aplicar e que promove o envolvimento do aluno para visualização dos procedimentos de enfermagem, o mesmo não possibilita o aprimoramento da prática clínica e é preciso a realização de momentos em laboratório para que a acadêmico desenvolver tais habilidades.

Revisão integrativa de literatura realizada no Brasil sobre estratégias educativas aplicadas no ensino de enfermagem sobre punção venosa infere que, apesar das tecnologias digitais disponibilizadas, o seu uso não anula a importância dos encontros presenciais. Os melhores resultados são alcançados quando o aluno recebe conteúdo teórico e práticas associadas ao simulador virtual ou outras tecnologias digitais. Assim, novas estratégias de aprendizagem que favoreçam a aquisição de conhecimentos e habilidades para prestação de cuidados seguros e eficientes que não gerem iatrogenias relacionadas à assistência de enfermagem devem ser implementadas na graduação com intuito de fomentar o desenvolvimento de competências para realização da punção venosa ${ }^{20}$.

No presente estudo, foi possível identificar que a maioria dos participantes nunca realizou PVP, o que corrobora com pesquisa realizada no Rio Grande do Norte, com participação de estudantes de cinco semestres do curso de enfermagem em que os resultados evidenciaram que os acadêmicos não possuem habilidades práticas para realização do procedimento ${ }^{21}$.

Para acadêmicos que não possuem experiência em PVP, obter conhecimento acerca da anatomia vascular, tornase fundamental para a execução do procedimento. Neste estudo, houve estatística significante $(p=0,000)$ sobre anatomia das veias. Esses achados corroboram com resultados encontrados em pesquisa no Ceará, com utilização de hipermídia, na qual obteve $14,9 \%$ de diferença nos números de acertos após o uso da tecnologia educativa sobre punção venosa. Acadêmicos de enfermagem acreditam que a melhora de conhecimentos e aprimoramento a respeito da anatomia da rede venosa favorece a redução de punções incorretas e complicações ${ }^{22,23}$.

Outra ação para evitar eventos adversos é mediante a realização de antissepsia com álcool a 70\% antes da punção para diminuir os patógenos cutâneos, o que torna essencial a adoção de métodos de aprendizado e educação contínua efetiva como a utilização de tecnologias. Desse modo, os achados de revisão sistemática com metanálise, realizada na Holanda, sobre a utilização de tecnologia audiovisual para ensino da punção venosa, mostraram que houve redução de $17,9 \%$ referente à contaminação, após a aplicação de intervenção tecnológica ${ }^{24}$.

A realização da punção com auxílio da inspeção e palpação, foi outra variável na qual houve melhora significante de acertos. A relevância de fato justifica-se pois os métodos propedêuticos tornam a realização do procedimento seguro e com maiores chances de sucesso, embora existam estratégias com maior recurso tecnológico como a punção guiada por ultrassonografia, que atribuem maior precisão ao procedimento e têm contribuído para menores números de tentativas de punção, como maior nível de satisfação dos pacientes ${ }^{25}$. 
Outro achado significativo foi a respeito da utilização do garrote que contribui para a congestão da circulação venosa local, o que torna as veias mais proeminentes e visíveis, deve ser aplicado acima do local de punção, no entanto não por tempo prolongado. Vale ressaltar, a necessidade de limpeza e desinfecção correta para promover a segurança do paciente e da equipe de profissionais, pois podem ser veículos reservatório e de transmissão de microrganismos multirresistentes, o que promove a contaminação cruzada.

Outra informação que possui melhora significante de acertos foi a administração de medicação em bolus com o uso do scalp. A utilização de cateter com agulhas de aço deve ser evitada para terapias intravenosas de longa duração, pois pode causar extravasamento dos medicamentos e irritação no vaso ${ }^{26}$. Assim, a adesão ao cuidado de evitar o uso de scalp favorece a redução de complicações e melhora a satisfação do paciente com a assistência de enfermagem ofertada, o que permite prática clínica livre de danos.

Vale ressaltar que o risco de desenvolver complicações locais e sistêmicas associadas à falha da PVP pode resultar em estadias hospitalares mais prolongadas e angústia para pacientes. As complicações mais frequentes infiltração, inchaço, obstrução, flebite, remoção acidental e outros. Assim, uma pesquisa realizada em Cingapura revelou que $67,6 \%$ dos cateteres são removidos por decorrente de alguma complicação ${ }^{27}$.

Com isso, é pertinente o uso de diversas estratégias para o ensino de PVP, para que, os acadêmicos de enfermagem compreendam a técnica, seus riscos e benefícios. Para transformar em acessível e dinâmica o processo de ensino-aprendizado, a incorporação de tecnologias torna-se uma ferramenta que proporciona o ambiente ativo para o aprendizado com diferentes métodos. Que vai de encontro ao estudo desenvolvido na Suécia, que afirma a respeito do uso de tecnologias no ensino, pois favorece a aprendizagem sobre a temática, que resulta na redução de intercorrências ou complicações durante os procedimentos de enfermagem, bem como garanti a segurança do paciente ${ }^{28}$.

O uso de tecnologia do tipo audiovisuais no processo de ensino-aprendizado é considerado estratégia eficaz para graduação em enfermagem, por colaborar para processo de participação protagonista do aluno para o aprendizado. Entretanto, estudo randomizado na Turquia, que utilizou plataforma de rede social digital para publicar o método de realização da administração de medicamentos não identificou diferença significante entre o uso do Instagram e o ensino em sala de aula ${ }^{29}$.

Ressalta-se, então, que as tecnologias oportunizam novos ambientes de aprendizados na área da enfermagem, de forma a contribuir na formação acadêmica e educação permanente ao fornecer subsídio interativo por meio de ferramentas audiovisuais que contribuem para o aprendizado. Entretanto, há obstáculos para seu desenvolvimento, como infraestrutura de informática e a necessidade de capacitações para doentes quando ao seu desenvolvimento e manuseio ${ }^{30}$.

\section{Limitação do estudo}

Esse estudo apresenta como limitação ter sido desenvolvido em universidade pública e os achados podem não refletir a realidade de outros cenários de ensino.

\section{CONCLUSÃO}

Houve melhora do conhecimento após a intervenção educativa em que a mediana de acertos no teste teórico passou de 15 para 16 com significância $(p=0,000)$. Verificou-se que, dos 20 itens, sete apresentaram aumento de acertos com significância estatística. Assim, o vídeo mostrou-se efetivo para auxiliar no ensino da enfermagem sobre a PVP.

Deve-se haver maior incentivo no emprego de vídeo educativo nas disciplinas práticas do curso de enfermagem com intuito de otimizar a aprendizagem dos estudantes. Sugere-se a realização de futuros estudos que visem a construção de tecnologias educativas sobre PVP tanto digitais como impressas e que esses recursos sejam comparados quanto à efetividade no ensino sobre a temática.

\section{REFERÊNCIAS}

1. Mermel LA. Short-term peripheral venous catheter-related bloodstream infections: a systematic review. Clinical Infectious Diseases. 2017 [cited 2020 June 15]; 65(10):1757-62. DOI: https://doi.org/10.1093/cid/cix562.

2. Alexandrou E, Ray-Barruel G, Carr PJ, Frost SA, Inwood S, Higgins N, et al. Use of short peripheral intravenous catheters: characteristics, management, and outcomes worldwide. J. Hosp. Med. 2018 [cited 2020 June 10]; 13(5). DOI: https://doi.org/10.12788/jhm.3039.

3. Braga LM, Parreira PM, Oliveira ASS, Mónico LDSM, Arreguy-Sena C, Henriques MA. Phlebitis and infiltration: vascular trauma associated with the peripheral venous catheter. Rev. Lat. Am. Enfermagem. 2018 [cited 2020 July 01]; 26:e3002. DOI: https://doi.org/10.1590/1518-8345.2377.3002.

4. Chavaglia SRR, Barbosa MH, Santos AS, Duarte RD, Contim D, OhI RIB. Didactic strategies identified by nursing students. Cogitare Enfermagem. 2018 [cited 2020 June 07]; 23(3):1-9. DOI: http://dx.doi.org/10.5380/ce.v23i3.53876. 
5. Tibes CM, Dias JD, Westin UM, Domingues AN, Zem-Mascarenhas SH, Évora YDM. Development of digital educational resources for nursing education. Rev Enferm Ufpe On Line. 2017 [cited 2020 June 15]; 11(3):1326-34. Available from: https://periodicos.ufpe.br/revistas/revistaenfermagem/article/viewFile/13972/16815

6. Hampton D, Pearce PF, Moser DK. Preferred Methods of Learning for Nursing Students in an On-Line Degree Program. J. Prof. Nurs. 2017 [cited 2020 June 10]; 33(1):27-37. DOI: https://doi.org/10.1016/j.profnurs.2016.08.004.

7. Silva LTC, Diniz FA, Gontijo TL, Machado RM, Cavalcante RB. Nursing students' perception on distance learning. Ciencia y Enfermeria. 2016 [cited 2020 June 09]; 22(2):129-39. DOI: https://doi.org/10.4067/S0717-95532016000200010.

8. Dalmolin A, Girardon-Perlinia NMO, Coppettia LC, Rossato GC, Gomes JS, Silva MEN. Educational video as a healthcare education resource for people with colostomy and their families. Rev. Gaúcha Enferm. 2016 [cited 2020 June 07]; 37:1-9. DOI: http://dx.doi.org/10.1590/1983-1447.2016.esp.68373.

9. Turaç N, Çalışkan N, Gülnar E. Comparison of mastery learning model and WhatsApp assisted learning in teaching psychomotor skills: a triangulation study. International Journal of Human Sciences. 2017 [cited 2020 June 08]; 14(3):2601-15. DOI: https://doi.org/10.14687/jhs.v14i3.4769.

10. Araujo PJA, Melo TCM. Literature review of audiovisual practices in Nursing education. Rev. Bras. Enferm. 2019 [cited 2020 June 08]; 72(1):190-9. DOI: http://dx.doi.org/10.1590/0034-7167-2017-0890.

11. Frota NM, Barros LM, Araújo TM, Lopes MVO, Almeida PC, Caetano JÁ. Validation of educational hypermedia about peripheral venipuncture. Texto contexto - enferm. 2015 [cited 2020 June 08]; 24(2):353-61. Available from: https://doi.org/10.1590/010407072015003480013.

12. Silva I, Ângelo J, Santos F, Lumini MJ, Martins T. Satisfaction and usability of an information and communications technology in nursing education: a pilot study. Rev. Enf. Ref. 2019 [cited 2020 June 08]; serIV (21):143-50. DOI: https://doi.org/10.12707/RIV19013.

13. Yang X, Xie RH, Chen S, Yu W, Liao Y, Krewski D, Wen SW Using Video Feedback Through Smartphone Instant Messaging in Fundamental Nursing Skills Teaching: Observational Study. JMIR Mhealth Uhealth. 2019 [cited 2020 June 09]; 7(9):e15386 DOI: https://doi.org/10.2196/15386.

14. Frota NM, Barros LM, Araújo TM, Caldini LN, Nascimento JC, Caetano JA. Construction of an educational technology for teaching about nursing on peripheral venipuncture. Rev. Gaúcha Enferm. 2013 [cited 2020 June 10]; 34(2):29-36. DOI: https://doi.org/10.1590/S1983-14472013000200004.

15. Pereira KC, Cogo ALP, Silva APSS. Critical analysis of peripheral catheter venipuncture videos available on Youtube. Reme: Revista Mineira de Enfermagem. 2016 [cited 2020 June 10]; 20:97076. Available from: https://www.lume.ufrgs.br/handle/10183/150364.

16. Castro FSF, Dias DMV, Higarashi IH, Scochi, CGS, Fonseca, LMM. Evaluation of digital educational student technology interaction in neonatal nursing. Rev. esc. enferm. USP. 2015 [cited 2020 June 13]; 49(1):114-21. DOI: https://doi.org/10.1590/s0080623420150000100015.

17. Souza-Junior VD, Mendes IAC, Tori R, Marques LP, Mashuda FKK, Hirano LAF et al. VIDA-Nursing v1.0: immersive virtual reality in vacuum blood collection among adults. Rev. Latino-Am. Enfermagem. 2020 [cited 2020 June 01]; 28:e3263. DOI: https://doi.org/10.1590/1518-8345.3685.3263.

18. MacLean S, Geddes F, Kelly M, Della P. Video Reflection in Discharge Communication Skills Training With Simulated Patients: a qualitative study of nursing students' perceptions. Clinical Simulation in Nursing. 2019 [cited 2020 July 01]; 28:15-24. DOI: https://doi.org/10.1016/j.ecns.2018.12.006.

19. Devi B, Khandelwal B, Das M. Comparison of the Effectiveness of Video-assisted Teaching Program and Traditional Demonstration on Nursing Students Learning Skills of Performing Obstetrical Palpation. Iran J. Nurs. Midwifery Res. 2019 [cited 2020 June 14]; 2(2):118-23. DOI: https://doi.org/10.4103/ijnmr.IJNMR_35_18.

20. Souza-Junior VD, Mendes IAC, Marchi-Alves LM, Jackman D, Wilson-Keates B, \& Godoy S. Peripheral Venipuncture Education Strategies for Nursing Students: An Integrative Literature Review. Journal of Infusion Nursing. 2020 [cited 2020 June 14]; 43(1):24-32. DOI: https://doi.org/10.1097/NAN.0000000000000351.

21. Melo GSM, Tibúrcio MP, Freitas CCS, Vasconcelos QLDAQ, Costa IKF, Torres GV. Semiotics and semiology of Nursing: evaluation of undergraduate students' knowledge on procedures. Rev. Bras. Enferm. 2017 [cited 2020 July 02]; 70(2):249-56. DOI: https://doi.org/10.1590/0034-7167-2016-0417.

22. Frota NM, Galindo Neto NM, Barros LM, Pereira FGF, Melo GAA, Caetano JÁ. Hypermedia on peripheral venipuncture: effectiveness in teaching nursing students. Rev. Bras. Enferm. 2018 [cited 2020 June 14]; 71(6):2983-9. DOI: https://doi.org/10.1590/0034-7167-2017-0205.

23. Frota NM, Barros LM, Costa A, Santos ZM, \& Caetano JA. Educational hypermedia on peripheral venipuncture: the perspective of students of nursing. Cogitare Enfermagem. 2014 [cited 2020 June 14]; 19(4). DOI: https://doi.org/10.5380/ce.v19i4.35384.

24. Van Loon FHJ, Buise MP, Claassen JJF, Dierick-van Daele ATM, Bouwman ARA. Comparison of ultrasound guidance with palpation and direct visualisation for peripheral vein cannulation in adult patients: a systematic review and meta-analysis. Br. J. Anaesth. 2018 [cited 2020 June 15]; 121(2):358-66. DOI: https://doi.org/10.1016/j.bja.2018.04.047.

25. Simundic AM, Bölenius K, Cadamuro J, Church S, Cornes MP, van Dongen-Lases EC, et al. Joint EFLM-COLABIOCLI Recommendation for venous blood sampling. Clinical Chemistry and Laboratory Medicine (CCLM). 2018 [cited 2020 June 18]; 56(12):2015-38. DOI: https://doi.org/10.1515/cclm-2018-0602.

26. Camerini FG, Silva LD, Mira AJM. Nursing actions for safe medication administration: an integrative review. Revista de Pesquisa Cuidado é Fundamental Online. 2014 [cited 2020 June 19]; 6(4):1655-65. Available from: http://www.indexf.com/pesquisa/2014/61655.php 
27. Tan YH, Tai WL, Sim C, Ng HL. Optimising peripheral venous catheter usage in the general inpatient ward: a prospective observational study. J. Clin. Nurs. 2017 [cited 2020 July 10]; 26(1-2):133-9. DOI: https://doi.org/10.1111/jocn.13451.

28. Ahlin C, Klang-Söderkvist B, Johansson E, Björkholm M, \& Löfmark A. Assessing nursing students' knowledge and skills in performing venepuncture and inserting peripheral venous catheters. Nurse Educ. Pract. 2017 [cited 2020 July 12]; 23:8-14. DOI: https://doi.org/10.1016/j.nepr.2017.01.003.

29. Vicdan AK. Assessment of the effect of mobile-assisted education regarding intramuscular injection by using the Instagram app. Nursing Practice Today. 2020 [cited 2020 June 20]. DOI: https://doi.org/10.18502/npt.v7i1.2302.

30. Brito Gonçalves LB, Pinto AGA, Duavy SMP, Santos Faustino R, Alencar APA, \& Palácio MAV. The Use of Digital Information and Communication Technologies as an Educational Resource in Nursing Education. EaD em Foco. 2020 [cited 2020 June 21]; 10(1):21. DOI: https://doi.org/10.18264/eadf.v10i1.939. 